\title{
Les transferts de radioéléments dans la chaîne alimentaire, les enseignements de Tchernobyl*, le programme Ressac
}

\author{
A. GRAUBY ${ }^{\star \star}$ \\ (Manuscrit reçu le 28 octobre 1987)
}

\begin{abstract}
RÉSUMÉ
Bien qu'à l'origine les études de transfert des radioéléments dans la chaîne alimentaire aient été mises en route à la suite de l'accident de Windscale (1958), l'étude des mécanismes de transfert a été essentiellement liée aux cas des rejets contrôlés des installations du cycle du combustible nucléaire. Si certaines données sur les processus de concentration dans les chaines biologiques sont utilisables pour les situations accidentelles, il apparaît, après l'accident de Tchernobyl, qu'un certain nombre de données doivent être revues tant au niveau de la modélisation des transferts que des facteurs de transfert eux-mêmes. Parmi les programmes français développés dans le domaine de l'impact des accidents, le programme RESSAC est bâti autour de trois préoccupations principales: mise au point de techniques de réhabilitation; mise au point d'un modèle prévisionnel et acquisition des principaux paramètres du modèle; acquisition de données d'ordre cartographique pouvant aboutir à la hiérarchisation des zones à traiter.
\end{abstract}

\section{ABSTRACT}

Studies on radionuclide transfers into the food chain were first initiated following the Windscale accident (1958); however, investigations on transfer kinetics have been mainly concerned with the case of controlled releases from nuclear fuel cycle plants. If some data on concentration processes in biological chains can be used in accidental conditions, the Chernobyl accident has demonstrated that some of them must be revised as concerns both transfer modeling and transfer factors. Among french programs developed in the field of the impact of accidents, the RESSAC program is built around three main concerns: development of reclamation techniques, development of a predictive model and acquisition of the main parameters of the model, acquisition of cartographic data, in order to have a scale of the areas to be cleaned.

Bien qu'à l'origine les études de transfert des radioéléments dans la chaîne alimentaire aient été mises en route à la suite de l'accident de Windscale (1958), l'étude des mécanismes de transfert a été essentiellement liée dans le passé aux cas des rejets contrôlés des installations du cycle du combustible nucléaire.

* Communication présentée lors du congrès franco-italien SFRP - Associazione italiana di protezione contro le radiazioni, Castelgandolfo (Italie), 12-13 octobre 1987.

** Commissariat à l'énergie atomique, Institut de protection et de sûreté nucléaire, DERS/ Service d'études et de recherche sur l'environnement, 13108 Saint-Paul-lez-Durance Cedex 147. 
Ces études, bien que nombreuses, n'ont peut-être pas toujours répondu aux demandes de ceux qui avaient la responsabilité de l'évaluation du risque c'est-à-dire du calcul de la dose reçue. Au prix d'une démarche conservatrice, ces facteurs de transfert obtenus expérimentalement ou in situ ont permis de cerner, pour un assez grand nombre de radionucléides, les limites de dose en fonction des rejets des différentes installations nucléaires. Certains ont même pensé que la radioécologie avait définitivement répondu aux questions soulevées par les radioprotectionistes au point de considérer cette discipline comme arrivant au terme de son existence.

Heureusement, quelques scientifiques avaient attiré l'attention, il y a cinq ans environ, sur les insuffisances en matière de connaissance sur les transferts dans ces chaînes alimentaires dans certains cas particuliers, notamment dans le cas des situations accidentelles. En effet, si certaines données sur les processus de concentration dans les chaînes biologiques sont parfaitement utilisables pour les situations accidentelles, il apparaît maintenant, après les premières observations effectuées in situ après l'accident de Tchernobyl, qu'un nombre de données doivent être revues tant au niveau de la modélisation des transferts que des facteurs de transfert eux-mêmes comme, par exemple, dans le cas de l'absorption foliaire.

Aujourd'hui, on mesure davantage la nécessité de ces programmes et les premiers résultats obtenus depuis cinq ans sont utiles pour tenter de comprendre et d'utiliser les observations faites dans l'environnement proche ou lointain de Tchernobyl. De plus, ils sont un argument pour témoigner de l'esprit de prévoyance et d'anticipation des autorités en matière de radioprotection.

Parmi les programmes français développés dans le domaine de l'évolution de l'impact des accidents, nous nous proposons de vous présenter le programme RESSAC. Le thème de cette étude est la mise au point de techniques de réhabilitation d'un environnement, notamment des sols et des nappes, après un accident nucléaire sévère qui entraîne des dépôts conséquents de radioactivité dans un rayon de 10 à $50 \mathrm{~km}$ autour de l'installation. Le programme RESSAC est bâti autour de trois préoccupations principales: mise au point de techniques de réhabilitation; mise au point d'un modèle prévisionnel et acquisition des principaux paramètres du modèle; acquisition de données d'ordre cartographique pouvant aboutir à la hiérarchisation des zones à traiter.

\section{TECHNIQUE DE RÉHABILITATION}

Une technique de réhabilitation est définie par son efficacité, sa vitesse et son coût d'emploi, le risque encouru par le personnel d'intervention et le type de déchets produits.

Nous avons choisi de ne baser nos études que sur du matériel pré-existant. La disponibilité du matériel requis, sa vitesse et son coût d'emploi sont donc aisément calculables, pour chacune des régions concernées. Nos études ne portent donc que sur l'efficacité d'enlèvement de la contamination, sur le type de déchets produits, étant entendu que celui-ci doit être 
aisément confinable et contrôlable, et sur la meilleure caractérisation possible de l'ambiance de travail des intervenants (exposition interne, exposition externe). Les expériences ne pouvant, bien entendu, avoir lieu qu'en plein champ (problèmes d'échelle), RESSAC a donc acquis les moyens culturaux pour pouvoir réaliser les couvertures végétales les plus fréquemment rencontrées en France. Le dépôt d'aérosol est simulé par l'émission, au-dessus des cultures, d'un nuage d'uranine ${ }^{(1)}$ qui arrive sec au niveau du sol, avec un diamètre moyen en masse de 1,5 $\mu \mathrm{m}$. RESSAC a également testé des procédés de décontamination des sols agricoles par épandage de mousses polyuréthanes à cellules fermées ou de peintures vinyliques. Les essais sur taillis et forêts devraient débuter courant 1988; l'ensemble des essais des principales techniques de réhabilitation devrait être achevé fin 1990.

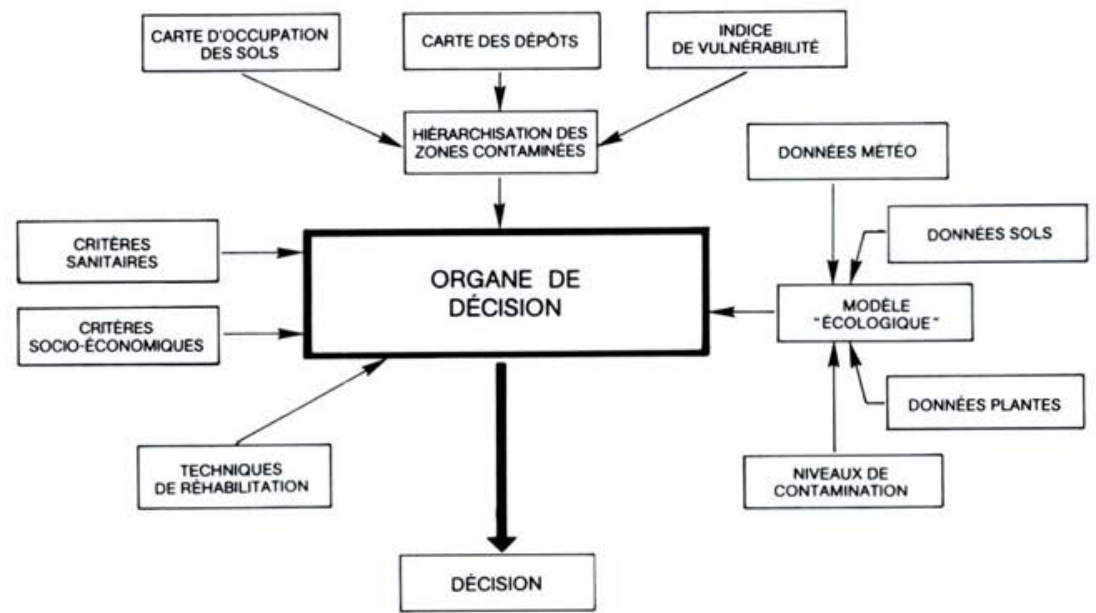

Stratégie de la réhabilitation.

\section{MOdÉLISATION DE LA DYNAMIQUE DES RADIOÉLÉMENTS}

Cette partie du projet a pour objet la construction de modèles simples et rapides d'emploi, pouvant être utilisés dans tous les PC de crise sur microordinateur. Ce point du programme a été abordé d'une part par la voie analytique, d'autre part au moyen d'expériences globales.

La phase analytique, en cours actuellement, traite de la dynamique des trois principaux radioéléments pris en compte par RESSAC, à savoir $\mathrm{Cs}, \mathrm{Sr}$ et Ru. Le principal problème est posé par l'évolution dans le sol des formes chimiques initiales. Cette évolution s'accompagne bien évidemment d'une évolution du comportement dans le milieu naturel; or, tous les modèles décrivant une dynamique d'éléments sont descriptifs de situations chimiquement invariantes. Pour ce qui concerne la dispersion des radioéléments

(1) Colorant dérivé de la fluorescéine. 
dans les sols, un second problème apparaît, en ce sens que nous devons travailler en milieu insaturé et que, là encore, il n'existe pas de modélisation performante.

Les études de transfert sol-plante sont menées à partir de l'étude des fractions biodisponibles contenues dans les sols. L'ensemble de ces essais en laboratoire sera achevé par une campagne de deux ans d'études sur cases lysimétriques ${ }^{(1)}$, permettant de simuler avec un maximum de vraisemblance l'ensemble des conditions climatiques et de gestion des sols (amendement, assolement,...). Ce type d'essais sera mené sur quatre types de sol, choisis à partir de l'étude analytique, comme représentatifs de l'ensemble des sols rencontrés au voisinage des réacteurs français à eau sous pression. Les lysimètres seront placés dans un bâtiment adéquat. L'exploitation devrait commencer en 1990 et la première campagne d'essais doit s'achever en 1992-93.

\section{ENQUÊTES PRÉALABLES}

Pour savoir à quel type de sol et de couverture végétale RESSAC devait s'intéresser, il a été nécessaire de lancer une enquête sur l'ensemble des vingt et un sites électronucléaires français. Cette enquête avait pour objectif de fournir la liste, classée par ordre d'importance surfacique, des types de sols et de cultures rencontrés dans un rayon de $10 \mathrm{~km}$. Cette démarche conduisait naturellement à la construction de cartes, du moins en ce qui concerne l'occupation des sols, de même en ce qui concerne la vulnérabilité des nappes phréatiques.

Ainsi, l'objectif général de RESSAC est de fournir un outil d'aide à la décision afin de réduire à son minimum technique le détriment causé à l'environnement par un accident majeur sur une centrale électronucléaire (à eau sous pression dans un premier temps). La réalisation de cet objectif est envisagée en trois volets: élaboration d'une stratégie d'intervention; choix de moyens tactiques; construction d'un outil permettant d'adapter l'ensemble aux particularités régionales (modèle mathématique).

Le récent accident de Tchernobyl a montré d'une part que les critères d'évaluation du détriment n'étaient pas uniquement des critères sanitaires, mais que s'y superposaient souvent des considérations économiques et politiques; et d'autre part qu'il était nécessaire de disposer de techniques d'intervention ponctuelles, utilisables dans des délais relativement courts, afin de permettre l'accès aux zones proches de l'installation accidentée, où une intervention rapide est nécessaire pour réduire les conséquences irrémédiables.

Enfin, on a pu remarquer sur certains sols que la dynamique des radioéléments, et notamment du césium, n'était pas celle prévue par les modèles

(1) Case lysimétrique ou lysimètre: case étanche contenant des blocs de terre sur lesquels sont cultivées différentes plantes dans des conditions climatiques normales. Ces cases sont munies d'un fond recueillant les eaux de filtration. Elles permettent, pour différents types de sol, la mesure des valeurs caractéristiques de l'humidité du sol (capacité de rétention, point de flétrissement permanent). 
construits à partir des données relatives aux rejets contrôlés. Ainsi se trouvent confortées - a posteriori - les idées directrices des programmes accidents, à savoir:

- les connaissances acquises jusqu'à maintenant ne peuvent pas toutes être utilisées en cas d'accident nucléaire;

- l'environnement doit être considéré non pas comme une interface entre le rejet et l'homme, mais plutôt comme le support de notre économie, ce qui doit influencer notablement la stratégie de l'intervention.

En dernier lieu, considérant les surfaces touchées dès l'instant où l'on prend en compte ce critère, il devient impensable d'envisager l'utilisation systématique et exclusive de moyens spécifiques et de personnel spécialisé. II faut donc s'attacher à étudier des moyens et des méthodes utilisables à partir du matériel et des hommes disponibles sur place (entreprises agricoles et de travaux publics).

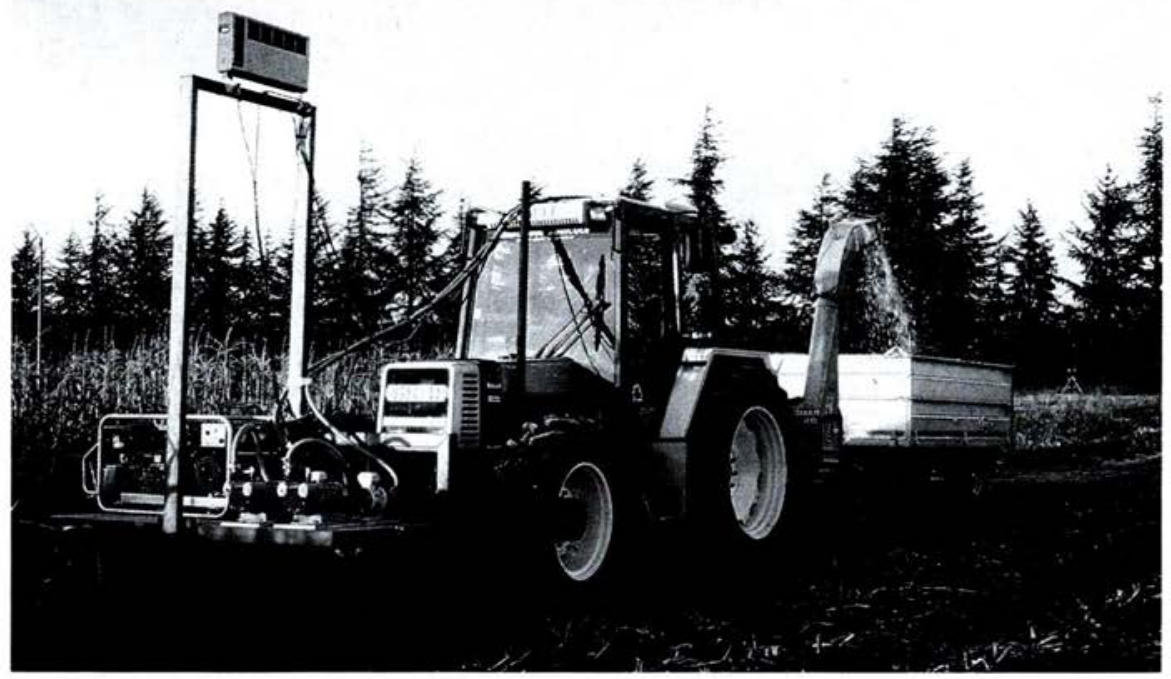

Fig. 1. - Essais d'ensilage.

\section{CONCLUSION}

Ainsi apparaît une situation nouvelle en matière de radioprotection, l'évaluation du risque économique et de ses conséquences sur les recommandations à donner aux autorités en matière de contremesures. Dans ce domaine, particulièrement important pour la radioécologie, de nouveaux programmes doivent être lancés à partir des données apportées par Tchernobyl. Ainsi, la CEE a proposé depuis quelques mois un programme spécifique sur les technologies agricoles et alimentaires qui peuvent modifier les niveaux radiologiques de ces produits pour permettre à la fois leur échange et leur consommation. 


\section{A. GRAUBY}

En France, nous entreprenons avec l'aide de la Communauté deux types d'études à partir des résultats du programme RESSAC pour définir des contremesures par fertilisation spécifique des sols. D'autre part, un programme de simulation des techniques agro-alimentaires a été mis en route qui reprend et prolonge des expériences que nous avions réalisées il y a plus de quinze ans sur différents matériaux agro-alimentaires, fruits, légumes et les techniques appropriées : fermentation, cuisson, biotechnologie, etc.

Là encore, Tchernobyl est un excellent moteur ; par exemple, les résultats soviétiques montrent que le lait impropre à la consommation l'est devenu par sa transformation en beurre. Des procédés de décontamination de ce même produit, notamment pour le césium, ont été mis au point dans différents pays européens.

Ainsi, si l'accident de Tchernobyl a conforté l'orientation des programmes en matière de protection de l'environnement vers les situations accidentelles, il a également apporté des informations particulièrement utiles. 